\title{
Tatort: Migration
}

Das Thema Einwanderung in der Krimireihe Tatort

\section{Christina Ortner}

Unter Berücksichtigung der Bedingungen für Integration und der Merkmale fiktionaler Fernsebinhalte fragt der Beitrag nach Möglichkeiten von Serien und Filmen, positiv auf den Integrationsprozess zu wirken. Vor diesem Hintergrund wird mit Hilfe eines Überblicks über sämtliche Tatort-Folgen zum Thema Migration sowie durch qualitative Inhaltsanalysen ausgewählter Episoden die Thematisierung von Migration in der Krimireibe Tatort beleuchtet. Dabei lässt sich feststellen, dass der Einwanderung insbesondere seit Ende der 80er Jabre erbebliche Bedeutung zukommt. Eine wesentliche Rolle spielen dabei die Sendeanstalten WDR, NDR und BR. Die fünf analysierten Tatort-Filme beleuchten das Thema Migration vielseitig, distanzieren sich deutlich von Ausländerfeindlichkeit und zeigen Beispiele für konfliktfreies Zusammenleben auf. Vereinzelt werden auch Kriterien für gesellschaftliche Zugebörigkeit hinterfragt. Obwobl Migrantenfiguren durchweg eher positive Eigenschaften aufweisen, werden stereotype Vorstellungen zum Teil bestärkt; darüber hinaus dominieren die deutschen Figuren maßgeblich die Erzählperspektive.

Schlüsselwörter: Migration, Einwanderung, Ausländer, Integration, Ausländerfeindlichkeit, Vorurteile, Tatort, Fernsehinhalte, Unterhaltung, qualitative Inhaltsanalyse

\section{Problemaufriss}

Derzeit leben in Deutschland rund 7,3 Mio. Menschen ohne deutsche Staatsbürgerschaft. Da Zuwanderung auch in Zukunft die Zusammensetzung der Population erheblich beeinflussen wird, muss Integration als wesentliche gesellschaftliche Aufgabe begriffen werden. Als Ort öffentlicher Diskussion spielen Medien dabei eine wichtige Rolle.

In der Kommunikationswissenschaft wird der Zusammenhang von Medien und Migration im Wesentlichen aus zwei Perspektiven betrachtet. Einerseits stellt sich die Frage, ob Medieninhalte als Verstärker von Vorurteilen und Fremdenhass wirken, andererseits steht insbesondere in den letzten Jahren die Bedeutung der Medien für den Integrationsprozess im Vordergrund. Obwohl zahlreiche Studien zu diesem Themenbereich vorliegen, ${ }^{1}$ mangelt es nach wie vor an wissenschaftlich fundierten Erkenntnissen und ausgereiften theoretischen Konzepten. Selbst im am besten untersuchten Bereich der Medieninhalte lassen sich Forschungsdefizite feststellen: Im Gegensatz zu den USA konzentrieren sich inhaltsanalytische Arbeiten in Deutschland vorwiegend auf die Präsentation ethnischer Minderheiten in Printmedien (Delgado, 1972; Merten, 1986; Ruhrmann/Kollmer, 1987; Jäger, 2000; Meißner/Ruhrmann, 2000). ${ }^{2}$

Obwohl das Fernsehen nach dem Hörfunk das meistgenutzte Medium ist (Gerhards/ Klingler, 2005: 558), liegen zu TV-Inhalten nur wenige Studien vor. Diese widmen sich

* Dank gilt Ingrid Paus-Hasebrink für die wissenschaftliche Begleitung der Arbeit im Rahmen der Abteilung für Audiovisuelle Kommunikation am Fachbereich Kommunikationswissenschaft der Universität Salzburg.

1 Geißler und Pöttker (2005: 391) zählten über 1000 Arbeiten, darunter 90 Bücher.

2 Einen ausführlichen Überblick über den Forschungsstand lieferte zuletzt Müller (2005). 
fast ausschließlich Nachrichten- und Informationssendungen (Kühne-Scholand, 1987; Brosius/Esser, 1995; Weiß et al., 1995; Ruhrmann et al., 2006); vereinzelt werden auch Sport, (Daily-)Talks oder Shows berücksichtigt (Schorb et al., 2003; Krüger/Simon, 2005). Hinsichtlich fiktionaler Inhalte sind vor allem eine Arbeit von Güven (1996) zu Stereotypen in Serien, ein Beitrag von Dörner (2000) zur Integrationsfunktion von Kinofilmen, ein weiterer von Bulut (2000) zum Bild fremder Frauen in Film und TV sowie Thieles (2005) Promotion zu Flucht, Asyl und Einwanderung im Fernsehen interessant.

Da die Programmsparte Fiktion in Untersuchungen zu Medien und Migration bisher also weitgehend vernachlässigt wurde, widmet sich der vorliegende Beitrag der Thematisierung von Migration in einem der ältesten deutschen fiktionalen TV-Angebote: der Krimireihe Tatort. Seit der Erstausstrahlung 1970 erfreut sich die Reihe enormen Zuspruchs und auch heute zählen Tatort-Filme noch zu den quotenstärksten fiktionalen TV-Sendungen in Deutschland (Darschin/Gerhard, 2004: 147). In wissenschaftlichen Arbeiten wurde der Tatort bisher vorwiegend im Kontext der deutschen Krimi- und Seriengeschichte behandelt (zuletzt Wehn, 2002; Brück et al., 2003). Zudem gehen einige Arbeiten am Beispiel einzelner Tatort-Folgen allgemeinen Fragestellungen audiovisueller Kommunikation nach. ${ }^{3}$

\section{Das integrationsfördernde Potenzial fiktionaler Fernsehinhalte}

$\mathrm{Da}$ aus Mangel an ausgereiften Ansätzen nicht auf eine fundierte theoretische Basis zurückgegriffen werden kann, bildet die Verortung von Serien, Filmen und Reihen in der Debatte Medien und Migration die Grundlage für das Untersuchungskonzept. Unter Berücksichtigung der Voraussetzungen für Integration und der Besonderheiten der Darstellungsform werden dabei integrationsfördernde Potenziale fiktionaler Fernsehinhalte diskutiert.

Der Begriff Integration wird in der Soziologie in zwei grundsätzlichen Bedeutungen verwendet: Erstens wird Integration als Zusammenhalt einer Gesellschaft verstanden, die trotz der Heterogenität ihrer Angehörigen als Einheit wahrgenommen wird. Zweitens meint Integration die Aufnahme von Nichtzugehörigen mit dem Ergebnis, dass sie zu „anerkannten Mitgliedern der aufnehmenden Gesellschaft werden“ (Volf/Bauböck, 2001: 14). Bauböck ${ }^{4}$ spricht von einem „Prozess der wechselseitigen Anpassung und Veränderung" (ebd.), ${ }^{5}$ in dem sowohl Migranten als auch die Aufnahmegesellschaft Leistungen erbringen müssen. Medien spielen dabei - besonders in Bezug auf strukturelle Herausforderungen an das Einwanderungsland - zweifelsohne eine begrenzte Rolle. Bedenkt man aber, dass die Bedingungen, unter denen Integration stattfindet, vom Selbstverständnis der Aufnahmegesellschaft abhängen und Akzeptanz kultureller

3 Bauer (1992) analysierte die Integration von Realität in der Fernsehunterhaltung u. a. am Beispiel einer Tatort-Folge. Süss (1993) ging ebenfalls anhand eines Tatort-Films Bedeutungsdifferenzen zwischen der Intention des Autors, dem Film als Medienprodukt und der Sichtweise junger Rezipienten nach. Kade (1996) widmete sich narrativen Konstruktionen von Biographien in Tatort und Polizeiruf 110 und Schnake (2000) beleuchtete die gezielte Verbindung von Unterhaltung und Information in Klinik unter Palmen und Tatort.

4 Der betreffende Band wurde von Volf und Bauböck gemeinsam verfasst und besteht aus zwei zusammengehörigen Teilen, die mit dem Namen des jeweiligen Autors gekennzeichnet sind.

5 Ähnliche Formulierungen finden sich auch bei Geißler (2000). 
Verschiedenheit notwendig für erfolgreiche Eingliederung ist, lassen sich mediale Einflüsse kaum leugnen.

Mit Abstand am häufigsten diskutiert wurde in diesem Zusammenhang die Möglichkeit, Ansichten von Inländern zu verändern, stereotype Bilder zu durchbrechen und Vorurteile abzubauen. Schon Ende der 80er Jahre gingen zwei Studien von Eckhardt (1987) und Esser (1986) unabhängig voneinander der Frage nach, ob negative Bewertungen von Menschen anderer Herkunft durch mediale Bilder beeinflusst werden können. Während Eckhardt meint, „für Resignation geben die Ergebnisse keinen Anlass“ (1987: 316), kommt Esser zu dem Schluss, massenmediale Kommunikation habe auf die Veränderung ethnischer Vorurteile kaum Einfluss (Esser, 2000: 36) ${ }^{6}$. Der Grund für die widersprüchlichen Befunde liegt neben der unterschiedlichen theoretischen und methodischen Herangehensweise in der Komplexität der „Zusammenhänge und Wechselwirkungen zwischen Erfahrung und der Produktion von Urteilen, Verallgemeinerungen, Klischeebildern, ethnischen und nationalen Stereotypen und Rassismen" (Liebhart et al., 2002: 7), welche die Identifikation unterschiedlicher Einflussfaktoren erschwert. Ergebnisse der Wirkungsforschung sprechen eher für einen schwachen Einfluss medialer Inhalte auf bestehende Einstellungen (Schenk, 2002: 702; Brosius, 2003: 136; WeberMenges 2005: 153). Laut Berghaus haben Rezipienten zu als wichtig empfundenen Themen weitgehend beständige Ansichten, die „im direkten sozialen Umfeld gebildet [...] und in die Medienbotschaften hineinprojiziert" (1999: 195) werden.

Dennoch bilden Informationen bzw. Vorstellungen über Migranten, die aufgrund von mangelnden persönlichen Erfahrungen häufig medial vermittelt sind, die Versatzstücke, mit deren Hilfe Vorurteile geformt werden. Berücksichtigt werden sollte zudem, dass sich negative - wie auch positive - Bewertungen laut Erkenntnissen der Stereotypenforschung an verallgemeinernden Kategorisierungen festmachen. Die Voraussetzung für die Ausbildung von Vorurteilen gegenüber Migranten bildet die Einteilung aller in einer Gesellschaft lebenden Menschen in In- und Ausländer. Diese beruht auf einer Wahrnehmung, die Differenzen betont, Ähnlichkeiten oder gemeinsame Interessen vernachlässigt und dadurch Identifikation mit den „Anderen“ verhindert. Die Kriterien der Abgrenzung sind dabei individuell verschieden und hängen vom Selbstverständnis als Zugehöriger einer der beiden Gruppen ab. Was die Identität als Angehöriger einer Nation, Kultur oder Gesellschaft ausmacht, wird unter den Mitgliedern der Gruppe ausgehandelt. Diese Verständigung über Selbstverständnis und Zugehörigkeitsgrenzen der Aufnahmegesellschaft wird zu großen Teilen über die Medien ausgetragen. Laut Jarren kommt Medien die Aufgabe zu, „gesellschaftliche Selbstverständigungsdiskurse $\mathrm{zu}$ ermöglichen und zu führen" (2000: 22f.). In medialen Inhalten manifestieren sich darüber hinaus auch Vorstellungen von Angehörigen anderer Nationen. Klaus, Goldbeck und Kassel sprechen in diesem Zusammenhang von der „diskursiven Erschaffung des ,Eigenen' und des ,Fremden' durch die Sprache und die Bilder der Medien“ (2002: 287). Medien sind daher maßgeblich an Einschließungs- und Ausgrenzungsprozessen beteiligt und haben Auswirkungen auf die Konstruktion der Trennlinie zwischen Inund Ausländern.

Im Hinblick auf das Thema Migration müssen Medienprodukte immer im Kontext ihrer spezifischen Merkmale betrachtet werden. Fiktionale Angebote zeichnen sich in erster Linie dadurch aus, dass sie nicht den Anspruch erheben, reale Ereignisse wiederzugeben, sondern unterhaltende Geschichten erzählen. Dieses Unterhaltungsverspre-

6 Esser bezieht sich mit dieser Aussage aus einem Beitrag von 2000 auf die Ergebnisse seiner Studie von 1986. 
chen eröffnet ihnen den Spielraum, Themen langsamer zu entwickeln und ausführlicher zu behandeln, da die Zuseher das Interesse auch über einen längeren Zeitraum nicht verlieren. Zudem können Inhalte unabhängig von Kriterien der Nachrichtenauswahl ausgewählt und losgelöst von tatsächlichen Gegebenheiten aufbereitet werden.? Weil das Zusehen vordergründig Vergnügen bereitet, sind Zuseher laut Hickethier ${ }^{8}$ (1991: 55) auch eher bereit, sich auf Themen einzulassen, mit denen sie sich sonst kaum beschäftigen, wodurch „Zugangsbarrieren, die gerade in der Konfrontation mit fremden Verhaltensweisen und Werten liegen“ (ebd.) reduziert werden können. Ähnliche Effekte schreibt Mikos (1987: 14) der figurenorientierten Erzählstrategie zu, welche die Zuseher in die Position unterschiedlicher Figuren versetzt und so zur Auseinandersetzung mit deren Ansichten beitragen kann. Da fiktionale TV-Inhalte diese Perspektive auch bei öffentlichen Themen beibehalten und gesellschaftliche Entwicklungen in ihrer Auswirkung auf Einzelne darstellen (ebd.), können sie abstrakte Themen verständlich darstellen und emotionalisieren. Darüber hinaus bieten Serien ein „Angebot von Verhaltensweisen" (Hickethier 1991: 50), das - unabhängig davon, ob die Zuseher es befürworten, ablehnen oder einfach zur Kenntnis nehmen - einen Orientierungsrahmen für das eigene Handeln darstellen kann (ebd.: 14). Wesentlich ist dabei nach Hickethier (ebd.: 54) die Bandbreite verschiedener Verhaltensformen. Das spezifische Potenzial fiktionaler Fernsehinhalte kann also u. a. darin liegen, dass sie leichter als andere Darstellungsformen gegenseitige Umgangsweisen von Menschen unterschiedlicher Herkunft und Lösungsansätze für Konflikte darstellen können.

\section{Fragestellung und Forschungsdesign}

\subsection{Fragestellung}

Vor diesem Hintergrund beleuchtet der vorliegende Beitrag die Frage, wie sich das inhaltliche Angebot der Krimireihe Tatort zum Thema Migration darstellt. Zum einen wird untersucht, wie häufig Einwanderung seit Beginn der Reihe als Haupt- oder Nebenthema vorgekommen ist und welche inhaltlichen Aspekte im Zentrum stehen. Zum anderen wird anhand von ausgewählten Beispielen gezeigt, welche Umsetzungsformen der Thematik sich in Tatort-Filmen beobachten lassen.

Als wesentlicher Aspekt filmischer Umsetzung von Geschichten mit Migrationsbezug kann die Thematisierung von Fremdenfeindlichkeit betrachtet werden. Explizite Aussagen zu dieser Problematik lassen sich in fiktionalen TV-Angeboten an Stellungnahmen einzelner Figuren festmachen. Hinsichtlich einer Auseinandersetzung mit eigenen Einstellungs- und Handlungsmustern seitens der Rezipienten sind vor allem die

7 Fiktionalität hat allerdings auch die Erwartungshaltung zur Folge, dass „nicht notwendig Tatsachen geschildert werden“ (Rothmund et al., 2001: 87). Folgt man Rothmund, Schreier und Groeben, so muss dies jedoch keinen Verlust an Glaubwürdigkeit nach sich ziehen. In einem mehrdimensionalen Modell zu Realitäts-Fiktions-Unterscheidungen differenzieren die Autoren zwischen drei Ebenen der rezeptionsseitigen Einschätzung von Realitätsbezug (ebd.: 86). Da Urteile über den Wirklichkeitsbezug nicht auf allen drei Ebenen gleich ausfallen müssen (ebd.: 88), kann ein Film auf der Ebene der Werkkategorie als Fiktion eingestuft, auf der Ebene des Erfahrungsinhalts sowie des Erfahrungsmodus aber durchaus als lebens- und realitätsnah empfunden werden.

8 Hickethiers Aussagen beziehen sich, ebenso wie jene von Mikos, auf Serien. Da sich die Erzählweise von Serien, Reihen und Fernsehfilmen im Hinblick auf das Thema Migration stark ähnelt, werden diese Aussagen hier auf fiktionale TV-Inhalte ausgeweitet. 
Aussagen jener Figuren von Bedeutung, die sich für deutsche Zuseher in besonderem Maße als Identifikationsfiguren anbieten. Aber auch Stellungnahmen von Migrantenfiguren können zur Reflexion von Fremdenhass und seinen Folgen beitragen, da sie die Sichtweise der Betroffenen einbringen. Neben Figurenaussagen spielen auch die Charakterisierung vorurteilsbehafteter Figuren, ihre Entwicklung im Laufe der Geschichte sowie ihre Motive und Lebenshintergründe eine Rolle.

Eng im Zusammenhang mit potenziellen Einflüssen auf Vorurteile steht die Frage nach der Figurenzeichnung von Einwanderern; diese kann Rezipienten in ihren bestehenden Vorstellungen sowohl bestärken als auch in Frage stellen. Zu beachten ist einerseits, inwieweit Migrantenfiguren stereotypen Vorstellungen entsprechen, und andererseits, ob die Darstellung positive oder negative Assoziationen nahelegt. Diese können durch Eigenschaften oder Verhaltensweisen der Figuren ebenso ausgelöst werden wie durch eine enge Verknüpfung mit Kriminalität oder gesellschaftlichen Problemen. Von Bedeutung sind demzufolge auch die Rolle der Migranten im Kriminalfall und der thematische Kontext, in dem sie auftreten.

Zudem muss berücksichtigt werden, welche Bedeutung Figuren mit Migrationshintergrund im Vergleich zu deutschen Figuren zukommt. Stehen Einwanderer im Zentrum von massenattraktiven Angeboten wie Tatort, so kann dies zur Wahrnehmung von Migranten als Mitglieder der Gesellschaft und zur Auseinandersetzung mit ihren Ansichten, Verhaltensweisen und Lebenswelten beitragen. In diesem Zusammenhang stellt sich die Frage nach der Gewichtung der Figuren. Neben der Anzahl von Migrantenfiguren und der Häufigkeit ihrer Auftritte sind dabei vor allem das Ausmaß an Informationen über Privatleben, Alltag, berufliches Umfeld, Lebensgeschichte, soziale, kulturelle oder religiöse Hintergründe einzelner Figuren sowie die Nachvollziehbarkeit ihrer Handlungen durch Angabe von Motiven oder Einblicke in ihre Wahrnehmungsund Gefühlswelt von Bedeutung.

Erzählungen mit Migrationsbezug liegen darüber hinaus Auffassungen von gesellschaftlicher Zugehörigkeit zugrunde, d. h., sie enthalten implizite Aussagen darüber, wer warum zur akzeptierten Gruppe der Inländer oder zur ausgeschlossenen Gruppe der Ausländer zählt. In fiktionalen Fernsehangeboten drückt sich die Grenze zwischen diesen beiden Gruppen vorwiegend in Art und Ausmaß der Unterschiede zwischen Einwanderern und Deutschen aus. Stellen Migrantenfiguren eine homogene Gruppe dar, die sich stark von den Deutschen unterscheidet und problemlos abgrenzen lässt, kann die Vorstellung von einer Zweiteilung der Gesellschaft verstärkt werden. Ein Figurenrepertoire, das sowohl die tatsächliche Heterogenität unter Einwanderern als auch die Gemeinsamkeiten zwischen Migranten und Inländern abbildet, kann diese Trennung unterlaufen. Aussagen zu gesellschaftlicher Zugehörigkeit sind mitunter auch auf der Ebene des Dialogs angesiedelt. Aus diesem Grund gilt es zu beachten, woran Figuren in Tatort-Episoden die Zugehörigkeit zur deutschen Gesellschaft festmachen.

Hinsichtlich der Erzählweise von fiktionalen Fernsehinhalten stellt sich schließlich die Frage nach Modellen des gegenseitigen Umgangs von Figuren unterschiedlicher Herkunft in Tatort-Filmen. Sowohl grundlegende Einstellungs- und Verhaltensmuster gegenüber den jeweils Fremden als auch die Beziehungen untereinander können von Distanz, Ablehnung, Gewaltbereitschaft oder aber von Akzeptanz, beiderseitigem Respekt bis hin zu Freundschaft geprägt sein. 


\subsection{Methodische Vorgehensweise}

Um sowohl den Stellenwert des Themas Migration in der Krimireihe Tatort als auch Details der filmischen Umsetzung dokumentieren zu können, erfolgte die Analyse in zwei methodischen Schritten.

Im ersten Schritt wurde anhand eines systematischen Überblicks über sämtliche Tatort-Folgen untersucht, wie häufig das Oberthema Migration in der Reihe vorkommt und welche spezifischen Aspekte der Thematik dominieren. Die Grundgesamtheit umfasste alle 566 Tatort-Filme, die bis Ende 2003 produziert wurden. Daraus wurden jene Folgen eruiert, die einen Bezug zum Thema Einwanderung aufweisen, d. h. die die Einreise von Migranten, ihren Aufenthalt im Aufnahmeland bzw. damit verbundene Schwierigkeiten für Migranten und/oder Angehörige der Aufnahmegesellschaft ansprechen. Da dieses Kriterium bereits erfüllt ist, sobald eine Figur mit Migrationshintergrund auftritt, wurden Folgen, in denen das Migrationsthema im Zentrum steht, in einer eigenen Kategorie erfasst. Da eine Sichtung sämtlicher 566 Tatort-Filme kaum leistbar ist, wurde die Kategorisierung auf Basis von Inhaltsangaben durchgeführt, wobei zur Verminderung von Unschärfen pro Folge bis zu drei Beschreibungen aus unterschiedlichen Quellen herangezogen wurden. ${ }^{9}$ Ebenfalls auf Basis dieser Angaben wurde anschließend der thematische Fokus - pro Film ein Aspekt - aller 32 Filme mit zentralem Migrationsbezug ermittelt.

In einem zweiten Schritt wurde mit Hilfe qualitativer Inhaltsanalysen ausgewählter Episoden untersucht, wie Tatort-Filme das Thema Migration umsetzen. Aus den insgesamt 92 Migrationsfolgen wurden in einem mehrstufigen Verfahren die fünf Folgen Brandwunden (RB, 1998), Fetischzauber (NDR, 1996), In der Falle (BR, 1998), Kinder der Gewalt (WDR, 1999) und Reise ins Nichts (SR, 2002) ausgewählt. Folgende Kriterien wurden dabei berücksichtigt: a) Das Thema Migration ist für die Filme von zentraler Bedeutung. b) Die ermittelnden Kommissare weisen insgesamt mindestens zehn Folgen - darunter drei Migrationsfolgen - auf; so kommen nur Ermittler vor, die bekannt sind und deren Einstellung zu Migration für weitere Folgen relevant ist. c) Die Thematik der ausgewählten Filme überschneidet sich nicht zu stark; auf diese Weise ist thematische Vielfalt gewährleistet. d) Aus demselben Grund wurde pro Kommissar und Sendeanstalt nur eine Folge ausgesucht. ${ }^{10}$ Von jeder der fünf Folgen wurden ein Szenen- sowie ein Detailprotokoll erstellt. Ersteres gibt einen Überblick über Schauplätze, Handlung und Figuren, Letzteres dient der genauen Erfassung von Bild- und Toninformationen sowie Kameraeinstellungen sämtlicher Szenen mit Migrationsbezug.

Als Analyseinstrument wurde ein Leitfaden mit fünf Dimensionen entwickelt, die im Folgenden kurz beschrieben werden.

- Die Dimension ,Thematisierung von Vorurteilen und Fremdenhass' bezieht sich auf Stellungnahmen einzelner Figuren zu ablehnenden Einstellungen, Aussagen oder Verhaltensweisen und erfasst die Charakterisierung vorurteilsbelasteter Figuren.

- Die Kategorie ,Darstellung der Migrantenfiguren' zielt auf Aussehen, Kleidung, Sprache, Einstellungen, Verhaltensweisen und Charaktereigenschaften von Migran-

9 Es handelt sich um Internetseiten der Sendeanstalten, der Produktionsfirmen sowie um die Seite www.Tatort-Fundus.de, die u. a. von Mitarbeitern der Universität Halle aufgebaut wurde.

10 Dieses Verfahren hat zur Folge, dass alle ausgewählten Filme zwischen 1996 und 2002 produziert wurden. Da ein Querschnitt über mehr als drei Jahrzehnte Tatort-Produktion mit fünf Folgen nicht geleistet werden kann, wurde auf eine ausgeglichene Verteilung der Produktionsjahre verzichtet. 
tenfiguren und fragt danach, ob sie hinsichtlich dieser Aspekte gängigen Vorstellungen über Personen ihrer Nationalität entsprechen. Zudem wird erhoben, welche Rolle Migrantenfiguren im Kriminalfall einnehmen und in welchem thematischen Kontext sie auftreten.

- Für die Dimension, Gewichtung der Figuren' sind die Anzahl von Einwanderern, die Häufigkeit ihrer Auftritte, Art und Ausmaß von Informationen sowie Angabe möglicher Motive im Vergleich zu deutschen Figuren von Bedeutung.

- Die vierte Kategorie ,Vorstellungen von gesellschaftlicher Zugebörigkeit erfasst einerseits Figurenaussagen darüber, wer aus welchen Gründen als Mitglied der Gesellschaft angesehen wird, andererseits fragt sie nach Art und Ausmaß der Unterschiede zwischen Deutschen und Migranten.

- Ausschlaggebend für die letzte Kategorie, Modelle des Umgang von Deutschen und Migranten' sind Einstellungen und Verhaltensweisen der Figuren gegenüber Angehörigen der jeweils anderen Gruppe sowie Beziehungen zwischen Figuren unterschiedlicher Herkunft.

Entlang dieser Dimensionen wurden die Szenenprotokolle der fünf Filme ausgewertet und sämtliche Szenen mit Migrationsbezug auf Basis der Detailprotokolle analysiert.

\section{Ergebnisse}

\subsection{Der Stellenwert von Migration in der Krimireibe Tatort}

In den 37 Jahren Laufzeit von Tatort nimmt Einwanderung neben einer Reihe von anderen gesellschaftlich relevanten Themen einen wichtigen Stellenwert ein; dies trägt den sozialkritischen Ambitionen Rechnung, die von Beginn an zu den Eckpfeilern des Konzepts gehörten. ${ }^{11}$ Von den insgesamt 566 Tatort-Episoden wird in zumindest 92 - also in 16,3\% aller Folgen - Einwanderung thematisiert, in 32 davon steht das Migrationsthema im Kern des Films. Bedenkt man die große Bandbreite an Themenbereichen, die Tatort-Filme abdecken, so kommt Einwanderung mit diesem Anteil eine beachtliche Rolle zu.

In den Anfangsjahren der Reihe stehen jedoch noch andere Themen im Vordergrund. Erst 1975, fünf Jahre nach Sendestart, geht mit Tod im U-Babnschacht die erste Tatort-Folge mit zentralem Migrationsbezug auf Sendung. 1976 ermittelt Kommissar Veigl im Milieu von ausländischen Schwarzarbeitern; ansonsten treten Einwanderer in den Tatort-Filmen der 70er nur in Nebenrollen auf. Ab 1981 wird jedes Jahr zumindest eine Migrationsfolge produziert, zu einem wesentlichen Anstieg kommt es schließlich in den Jahren 1987 und 1988. Auch in diesen Tatort-Produktionen der späten 80er ist Migration jedoch selten zentrales Thema.

Ins Zentrum von Tatort-Folgen rücken Geschichten mit Migrationsbezug erst häufiger ab 1991. Da sich Tatort-Filme in dieser Zeit vermehrt mit dem Aspekt rechtsradikaler Gewalt beschäftigen, hängt die verstärkte Aufmerksamkeit für das Thema vermutlich mit den Anschlägen auf Migrantenwohnheime Anfang der 90er Jahre zusammen. In den darauf folgenden Jahren kommt es zu einer intensiven Thematisierung von Einwande-

11 Als Kern des Konzeptes wird häufig Wirklichkeitsorientierung genannt; Bauer spricht sogar von einem „dezidiert mimetische[n] Anspruch“ (1992: 100). Der zweite Eckpfeiler, den Vogt als „das ,Tatort'-Prinzip schlechthin“ (2004: 116) bezeichnet, lässt sich mit dem Begriff Regionalismus beschreiben. Als weitere wesentliche Komponente betrachten Brück und Viehoff (1998: 8) die Thematisierung aktueller Probleme des sozialen und politischen Lebens. 
rung und ihren Auswirkungen in Tatort, die 1996 und 1998 ihren Höhepunkt erreicht und anschließend auf mittlerem Niveau fortgesetzt wird. Insbesondere in den 90er Jahren leistet die Reihe also einen wesentlichen Beitrag zur medialen Auseinandersetzung mit Migration, indem sie ein Massenpublikum mit Geschichten rund um Einwanderung konfrontiert.

Abb. 1: Anzabl der Migrationsfolgen pro Produktionsjabr

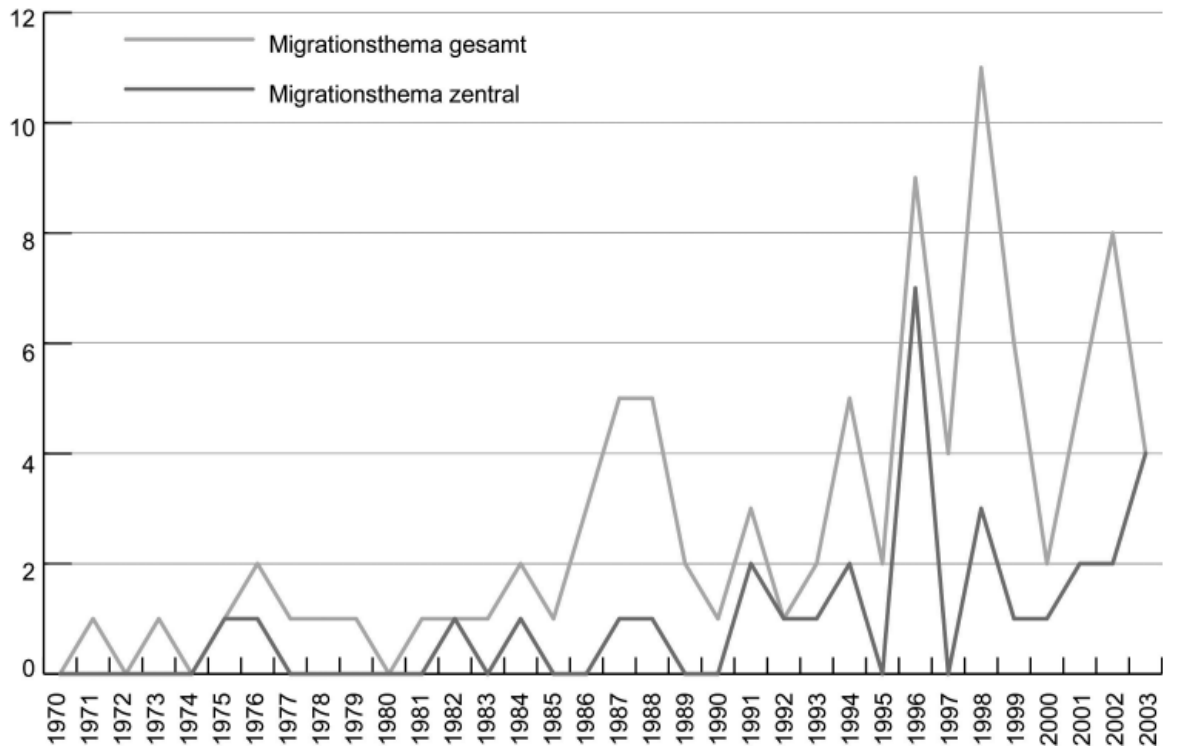

Die Bedeutung des Themas im Tatort spiegelt sich auch darin, dass sich jede der 13 beteiligten Sendeanstalten ${ }^{12}$ in ihren Folgen mit Einwanderung befasst. Mit 19 Filmen kommt der größte Anteil dem WDR zu, im überwiegenden Teil dieser Folgen (14) wird Einwanderung jedoch nur am Rande thematisiert. Der NDR liegt mit 14 Episoden hinter dem WDR, darauf folgt der BR mit elf Migrationsfolgen. Keine andere Sendeanstalt widmet sich dem Thema allerdings so intensiv, denn in sieben Tatort-Filmen des BR lässt sich Einwanderung als Hauptthema bezeichnen. Setzt man die Anzahl der Folgen mit Migrationbezug in Bezug zur Gesamtanzahl an Tatort-Folgen der jeweiligen Sender, ergibt sich ein etwas verschobenes Bild. Demnach weisen Radio Bremen sowie der schweizerische SF DRS den höchsten Anteil an Migrationsfolgen auf; diese Zahlen kommen aber nur dadurch zustande, dass beide Sender insgesamt sehr wenige Folgen produziert haben. Tatsächlich handelt es sich um lediglich je drei Episoden mit Migrationsbezug, also weniger als bei allen anderen Rundfunkanstalten. Lässt man RB und SF DRS beiseite, so liegt der SFB mit 18,6 \% gleichauf mit dem WDR an erster Stelle, gefolgt von MDR und SR mit je 18,2 \%, das Schlusslicht bilden HR und ORF. Hinsichtlich der Episoden mit

12 Durchgehend beteiligt waren BR, HR, NDR, RB, SFB, SR, WDR und ORF. SWF und SDR produzierten bis 1998 jeweils eigene Tatort-Folgen; anschließend wurden sie zum SWR zusammengeschlossen. Nach der Wiedervereinigung kam 1992 noch der MDR dazu. Zudem hat der SF DRS zwischen 1998 und 2001 zwölf Folgen produziert. 
zentralem Migrationsbezug kommt nach wie vor dem BR (11,3\%) die wichtigste Rolle zu. Auch SFB und SR weisen hier noch hohe Werte auf $(9,3 \%$ und 9,1\%).

\section{Abb. 2: Anzabl der Migrationsfolgen pro Sendeanstalt}

\begin{tabular}{lccccc}
\hline \multirow{2}{*}{$\begin{array}{l}\text { Sende- } \\
\text { anstalten }\end{array}$} & $\begin{array}{l}\text { Gesamtanzahl } \\
\text { der Folgen }\end{array}$ & Folgen mit Migrationsbezug & \multicolumn{2}{c}{$\begin{array}{l}\text { Folgen mit zentralem } \\
\text { Migrationsbezug }\end{array}$} \\
\cline { 3 - 6 } & & absolut & $\begin{array}{l}\text { in \% aller } \\
\text { Folgen }\end{array}$ & absolut & $\begin{array}{c}\text { in \% aller } \\
\text { Folgen }\end{array}$ \\
\hline WDR & 102 & 19 & 18,6 & 5 & 4,9 \\
NDR & 84 & 14 & 16,6 & 6 & 7,1 \\
BR & 62 & 11 & 17,7 & 7 & 11,3 \\
SFB & 54 & 10 & 18,6 & 5 & 9,3 \\
ORF & 52 & 5 & 9,6 & 2 & 3,9 \\
HR & 47 & 5 & 10,6 & 0 & 0 \\
MDR & 33 & 6 & 18,2 & 0 & 0 \\
SWR & 30 & 5 & 16,7 & 2 & 6,7 \\
SDR & 30 & 4 & 13,3 & 0 & 0 \\
SWF & 27 & 4 & 14,8 & 0 & 0 \\
SR & 22 & 4 & 18,2 & 2 & 9,1 \\
SF DRS & 12 & 3 & 25 & 1 & 8,3 \\
RB & 11 & 3 & 27,3 & 2 & 18,2 \\
Alle Sender & 566 & $\mathbf{9 2}$ & 16,3 & 32 & $\mathbf{5 , 6}$ \\
\hline
\end{tabular}

1 Die Prozentzahlen wurden auf eine Kommastelle gerundet.

2 Eine Folge mit Migrationsbezug (Folge 415) wurde von WDR und MDR gemeinsam produziert. Da sie für beide Sender gezählt, in der Gesamtanzahl aber nur einmal berücksichtigt wurde, stimmt die Summe nicht dem Wert 92 überein.

Wie zu erwarten, fällt die Reihung der Kommissare ähnlich aus wie die der dazugehörigen Sendeanstalten. Da die Anzahl der Filme mit Migrationsbezug erst seit 1987 und verstärkt seit 1991 ins Gewicht fällt, verteilen sich die 92 Migrationsfolgen vorwiegend auf die letzten beiden Kommissar-Generationen der größten Sendeanstalten. Schimanski (WDR) liegt dabei mit zehn Folgen an der Spitze, gefolgt von Stoever und Brockmöller (NDR) mit neun Episoden. Am intensivsten widmet sich das Münchner Team Batic und Leitmayr (BR) dem Thema Einwanderung: Mit acht Migrationsfolgen sind sie zwar nur an dritter Stelle, sechs ihrer Filme stellen das Thema aber in den Mittelpunkt der Krimihandlung. Zudem ist Batic selbst kroatischer Abstammung, was in den Folgen des BR wiederholt zu Diskussionen führt. Interessant ist auch Lena Odenthal (SWR), die seit 1996 mit dem italienischstämmigen Mario Kopper zusammenarbeitet und eine enge Freundschaft zur Tochter eines Senegalesen unterhält. Bemerkenswerterweise haben 44 der insgesamt 75 Tatort-Kommissare keinen einzigen Fall gelöst, bei dem das Thema Migration eine Rolle spielt. 
Abb. 3: Anzabl der Migrationsfolgen pro Kommissar/Team*

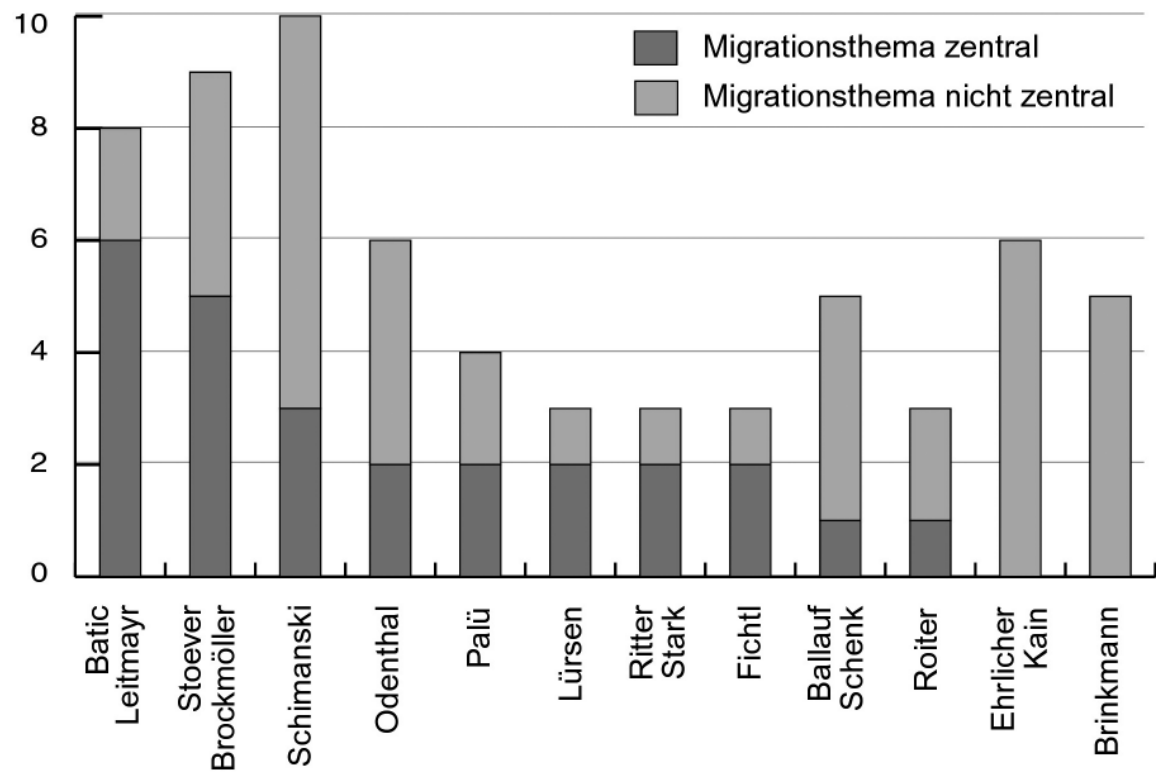

In diesem Diagramm wurden nur Kommissare erfasst, die mindestens drei Migrationsfolgen aufweisen.

Einwanderung wird im Tatort nicht nur häufig angesprochen, unter den Migrationsfolgen herrscht auch große thematische Vielfalt. Insgesamt decken die 32 Filme mit Migration als Hauptthema 14 unterschiedliche Teilaspekte ab. In sechs Filmen - und damit am häufigsten - wird ,internationaler Frauen- und Kinderhandel' thematisiert. Weitere wichtige Aspekte sind ,rechtsradikale Übergriffe auf Migranten' (4), ,illegale Beschäftigung ausländischer Arbeiter (4), ,Menschenschmuggel' (3) sowie ,Schwierigkeiten illegaler Einwanderer' (3). Geschichten in Zusammenhang mit, Gewalt in Schulen mit hohem Ausländeranteil', ,kriminellen ausländischen Kinderbanden' sowie ,politischem Mord an Migranten' stehen im Zentrum von je zwei Folgen. Der Gestaltungsspielraum fiktionaler Inhalte bei der Themenwahl wirkt sich im Tatort also dahingehend aus, dass Einwanderung in eine Vielzahl unterschiedlicher Zusammenhänge eingebettet wird. Auffälligerweise handelt es sich dabei vorwiegend um problembehaftete Kontexte. Dies birgt einerseits die Gefahr, Migration auf Problem- und Ausnahmesituationen zu reduzieren; eine differenzierte Darstellung von gesellschaftlichen Spannungen kann andererseits auch zur Auseinandersetzung mit bestehenden Problemen beitragen.

\subsection{Filmische Umsetzung von Migration in ausgewäblten Folgen}

Das vorangehende Kapitel hat gezeigt, dass Einwanderung häufig und vielseitig thematisiert wird. Anhand von Einzelanalysen der fünf ausgewählten Episoden wird im Folgenden beschrieben, wie einzelne Tatort-Episoden das Oberthema Migration filmisch umsetzen. 


\section{Brandwunden ${ }^{13}$ (RB 1998, Lürsen)}

Brandwunden erzählt die Geschichte einer türkischen Familie, deren Haus während ihres Urlaubs in Brand gesteckt wird. Dabei kommt ein junges türkisches Liebespaar ums Leben.

Als zentraler Aspekt des Films lässt sich ,Rechtsradikalismus‘ bezeichnen. Polizei, Opfer und Presse gehen bis kurz vor Schluss davon aus, dass nationalistische Motive hinter der Tat stecken. Im Laufe der Verhöre werden die Ermittler wiederholt mit fremdenfeindlichen Ansichten konfrontiert und zeigen einhellig Abneigung. Einerseits ist diese Reaktion dazu geeignet, gleich gesinnte Zuschauer in ihren Ansichten zu bekräftigen. Da eine Auseinandersetzung mit den Hintergründen ausbleibt, kann sie aber auch moralisierend wirken.

Die Figurenzeichnung der Migranten liefert sowohl Zusehern mit als auch ohne Vorbehalten Material zur Bestärkung ihrer Sichtweise. Der Großteil der türkischen Figuren wird differenziert charakterisiert und weist vorwiegend positive Eigenschaften auf; dessen ungeachtet fließen in die Figurenzeichnung auch klischeehafte Vorstellungen ein. Am deutlichsten trifft dies auf den gläubigen Moslem Altun zu. Er versucht, seine Tochter durch Schläge von ihrem Freund fernzuhalten und hält es für Allahs gerechte Strafe, als die beiden beim Brand ums Leben kommen. Bemerkenswert ist zudem, dass alle Migrantenfiguren im Zusammenhang mit schwerwiegenden Konflikten stehen.

Erzählt wird die Geschichte vorwiegend aus der Perspektive deutscher Figuren. Die größte Gruppe bilden Polizeibeamte, wobei Kommissarin Lürsen die wichtigste Rolle zukommt. Da sie ihre Meinungen, Emotionen und Schlussfolgerungen häufig artikuliert, ist ihr Verhalten gut nachvollziehbar. Lässt man die Polizisten beiseite, so liegt der Schwerpunkt eher bei den türkischen Figuren. Mit Hilfe von Dialogen zwischen den Migranten, Lürsen und ihren Mitarbeitern setzt sich der Film mit den Ansichten und der Lebenswelt der Einwanderer auseinander. Teils erklären die Einwanderer ihre Beweggründe auch selbst und ermöglichen so den Zusehern, die Ereignisse aus ihrem persönlichen Blickwinkel zu sehen. Die Deutschen haben hingegen in erster Linie die Funktion, die Krimihandlung voranzutreiben.

Auffassungen von gesellschaftlicher Zugehörigkeit sind in Brandwunden Thema einer heftigen Debatte zwischen Lürsen und ihrem Lebensgefährten. Dieser wirft ihr vor, sie weigere sich, Altun als gleichberechtigtes Mitglied der Gesellschaft anzuerkennen, weil seine Weltanschauung nicht ihren persönlichen Werten entspräche. Indem Lürsens persönliche Kriterien für gesellschaftliche Akzeptanz in Frage gestellt werden, besteht für die Zuseher die Möglichkeit, sich ihrer eigenen Konzepte bewusst zu werden. Die Figurenzeichnung in Brandwunden unterstützt nur bedingt Vorstellungen von zwei klar abgrenzbaren Gruppen, denn die Migrantenfiguren unterscheiden sich in ihren Lebensweisen und Einstellungen deutlich voneinander. Dennoch lässt der Film diese Leseweise $\mathrm{zu}$, denn die türkischstämmigen Figuren lassen sich durch ihr Aussehen problemlos von den Deutschen abgrenzen.

Eine Verschränkung von In- und Ausländern entsteht hingegen durch die teils engen Beziehungen zwischen Migranten und Deutschen. Familie Kazim verbindet mit ihrem Nachbarn Kruse eine langjährige Freundschaft, und die Kinder der beiden Familien sind sogar verlobt. Neben diesen positiven Modellen des Zusammenlebens finden sich

13 Der Film wurde 1998 produziert, erreichte bei der Erstausstrahlung am 26.4.1998 ca. sieben Mio. Zuseher und wurde seither sieben Mal wiederholt. (Tatort-Fundus, 2006) 
in Brandwunden auch konfliktbehaftete Umgangsformen bis hin zu gewalttätigen Auseinandersetzungen.

\section{Fetischzauber ${ }^{14}$ (NDR 1996, Stoever/Brockmöller)}

In Fetischzauber versuchen Stoever und Brockmöller gleich drei Todesfälle aufzuklären: den Mord an einem karibischen Einwanderer, den unerwarteten Selbstmord des Unternehmers Mevis und den tödlichen Autounfall seines Sohns.

Anders als in Brandwunden wird Ausländerhass in Fetischzauber nur am Rande thematisiert. Mit Ausnahme eines Dialogs, in dem ein Arbeiter seine farbigen Kollegen „schwarzes Pack“ schimpft, kommen keine fremdenfeindlichen Aussagen vor. Brockmöller weist diese Figur daraufhin vehement zurecht und macht deutlich, dass er Vorurteile gegenüber Migranten ablehnt. Dennoch gelingt es ihm selbst nicht, sich unvoreingenommen zu verhalten. Vielmehr reagieren beide Kommissare irritiert, verständnislos und ablehnend auf die karibischen Einwanderer.

Die Charakterisierung der Migranten liefert den Zusehern wenig Anlass, ablehnende Einstellungen Fremden gegenüber zu überdenken. Sie ist stark reduziert und macht sich an der Ausübung ihrer Kultur und Religion fest. Die beiden wichtigsten Migrantenfiguren sind Voodoopriester und treten überwiegend als Vertreter ihrer Gemeinde auf. Die religiösen Feste wirken durch Verhalten und Aussehen der Beteiligten sowie durch den Einsatz von Licht, Musik und Kameraführung bedrohlich. In einer Szene wird durch eine schnell geschnittene, eng verzahnte Montage von drei Schauplätzen sogar ein kausaler Zusammenhang zwischen einem Ritual und einem tödlichen Unfall suggeriert. Dennoch lässt sich die Figurenzeichnung der Migranten nicht als ausschließlich negativ bezeichnen, denn sie weisen auch zahlreiche positive Eigenschaften auf. In erster Linie kommt ihnen aber die Rolle der Verdächtigen zu.

Darüber hinaus gibt der Film den Rezipienten kaum Gelegenheit, die Geschehnisse aus der Sicht der Migranten zu betrachten, denn die Erzählung wird von der Perspektive der deutschen Figuren dominiert. Fast in jeder Szene tritt einer der beiden Ermittler auf, wodurch sich der Informationsstand der Zuseher beinahe lückenlos mit ihrem deckt. Lässt man Stoever und Brockmöller außer Acht, so liegt der Fokus immer noch deutlich auf den deutschen Figuren. Sie kommen nicht nur häufiger in Hauptrollen vor und weisen eine größere Anzahl von Auftritten auf, die Zuseher erfahren auch mehr über ihre Beziehungen, Probleme sowie über ihr familiäres und berufliches Umfeld.

Betrachtet man das Figurenrepertoire näher, so wird deutlich, dass Fetischzanber ein Bild von zwei entlang der Herkunft klar abgrenzbaren Gruppierungen mit getrennten Lebenswelten vermittelt, das wenig dazu geeignet ist, die Wahrnehmung von Einwanderern als Teil des gesellschaftlichen Alltags zu fördern. Die Migranten stellen eine homogene Gruppe dar, die sich stark von den Deutschen unterscheidet. Um ihre Religion ungestört ausüben zu können, schaffen sie eine kleine Teilgesellschaft, in der andere Regeln und Werte gelten. Der Großteil der Figuren ist je nach Herkunft einem bestimmtem Schauplatz zugeordnet, und mit Ausnahme der Kommissare treten deutsche Figuren kaum zusammen mit Einwanderern auf.

Aus diesem Grund werden in Fetischzauber kaum positive Modelle des Zusammenlebens von Menschen unterschiedlicher Herkunft gezeigt. In den seltenen Fällen, wo sich die Welten von In- und Ausländern überschneiden, kommt es zu konfliktbeladenen

14 Bei der Erstsendung am 5.5.1996 erreichte Fetischzauber neun Mio. Zuseher und damit einen Marktanteil von 27,08 \%. Seither wurde die Folge achtmal wiederholt. (Tatort-Fundus, 2006) 
Beziehungen. Auch der Umgang der Kommissare mit den Einwanderern stellt sich als schwierig dar.

\section{In der Falle ${ }^{15}$ (BR 1998, Batic/Leitmayr)}

In der Falle erzählt das Schicksal der jungen Türkin Bengi, die nach Jahren legalen Aufenthalts abgeschoben wird, illegal wieder einreist und Zeugin eines Mordes wird.

Wie in Fetischzauber wird auch in dieser Folge Fremdenfeindlichkeit kaum thematisiert, diskriminierende Ansichten und Verhaltensweisen kommen aber vor. Ein Beamter der Fremdenbehörde behandelt Einwanderer beispielsweise respektlos, ein Zeuge verbreitet Klischees über das türkische Volk, und zwei Polizeibeamte sprechen abfällig von „anatolischer Schwester“ und „phantasielosem Jugo“. In den meisten Fällen distanzieren sich die Kommissare von diesen Aussagen.

Die Charakterisierung der Migrantenfiguren dieses Films liefert Vorurteilen wenig Nahrung. Abgesehen von zwei Nebenfiguren entsprechen sie in keiner Weise gängigen Vorstellungen; zudem weisen die meisten von ihnen erstrebenswerte Eigenschaften auf und sind somit geeignet, Zuseher in positiven Einstellungen gegenüber Migranten zu bestärken. Eine Figur stellt sogar einen expliziten Bruch mit Klischees dar und bietet dadurch Anlass zur Reflektion: Es handelt sich um eine Putzfrau, die im ersten Moment in Aussehen, Sprache und Verhalten dem Bild einer unselbstständigen gläubigen Türkin ohne Deutschkenntnisse entspricht. Tatsächlich versteckt sich dahinter eine attraktive Frau Anfang 20, die in Deutschland aufgewachsen ist und sich während der Arbeit verkleidet, um nicht von Männern belästigt zu werden.

In der Falle liefert den Zusehern auch Einsichten in die Lebenssituationen der Einwanderer, denn Figuren mit Migrationshintergrund stehen im Mittelpunkt der Erzählung. Insbesondere gilt dies für Bengi und ihre Schwester. Zahlreiche Szenen liefern Auskunft über ihre Vergangenheit, Zukunftspläne und alltäglichen Probleme. Ihr Schicksal wird vor allem dadurch nachvollziehbar, dass sie stark über Emotionen und Beziehungen charakterisiert werden. Auch mit dem türkischstämmigen Täter Sinan setzt sich der Film ausführlich auseinander. Wiederholt werden seine Motivationen vor dem Hintergrund seiner Lebensgeschichte diskutiert. Da Sinans Unschuld bis kurz vor Ende bewiesen scheint, können sich die Zuschauer ohne Vorbehalte auf die Figur einlassen.

Mit Kriterien für gesellschaftliche Zugehörigkeit setzt sich In der Falle nicht explizit auseinander. Unabhängig von Staatszugehörigkeit und Aufenthaltsstatus sind Migranten aber auf vielfältige Weise in den gesellschaftlichen Alltag eingebunden. Sie absolvieren in Deutschland ihre Ausbildung, arbeiten in zentralen Positionen deutscher Firmen oder haben sich eigene Unternehmen aufgebaut. Die meisten von ihnen leben schon lange in Deutschland, betrachten dieses Land als ihre Heimat und planen hier ihre Zukunft. Da sich die Migranten stark untereinander, aber nur wenig von den Deutschen unterscheiden, unterwandert die Figurenzeichnung die Vorstellung von einer homogenen Gruppe der Inländer, die sich klar von Ausländern unterscheiden lässt.

Darüber hinaus bietet der Film ein breites Spektrum an möglichen Umgangsweisen zwischen Menschen unterschiedlicher Herkunft. Die Verhaltensweisen deutscher Figuren, zu denen die Rezipienten ihre eigenen Handlungskonzepte in Bezug setzen können, reichen von Ablehnung über Verständnis und Solidarität bis hin zu Freundschaft.

15 7,61 Mio. Menschen sahen In der Falle bei der Erstaussendung am 1.3.1998 (Marktanteil $20,05 \%$ ). Seither wurde die Folge siebenmal wiederholt. (Tatort-Fundus, 2006) 


\section{Kinder der Gewalt ${ }^{16}$ (WDR 1999, Ballauf/Schenk)}

In Kinder der Gewalt wird ein zehnjähriger Junge erschossen auf der Schultoilette gefunden. Die Ermittlungen erweisen sich als schwierig, denn der Schulalltag ist von der Feindschaft zwischen türkischen und deutschen Kindern geprägt.

Fremdenfeindlichkeit ist folglich ein zentrales Thema des Films, zu dem die Kommissare wiederholt Stellung beziehen. Als einziger Film setzt sich Kinder der Gewalt auch mit den Hintergründen der vorurteilsbelasteten Figuren auseinander. Die meisten Schüler leben in ärmlichen Verhältnissen, bekommen von ihren Eltern kaum Aufmerksamkeit und leiden an einem Mangel an Anerkennung, Wertschätzung und Perspektiven. Ganz nach dem Vorbild ihrer Eltern geben die Kinder den ,Türken' die Schuld dafür. Wiederholt ziehen die Kommissare diese Umstände als Erklärung für die Gewalt in der Schule heran. Dadurch wird deutlich, dass Ausländerhass im persönlichen Umfeld erlernt und kulturelle Konflikte durch soziale Bedingungen mitbestimmt werden.

Im Vergleich zu den deutschen Schülern erfahren die Zuschauer wenig über die Lebenssituation der türkischstämmigen Kinder. Die Figurenzeichnung der Migranten ist stark reduziert; sie bleiben bis zum Ende des Films austauschbar. Zudem entsprechen sie gängigen Bildern von türkischen Jugendlichen, sind an Prügeleien und kriminellen Delikten beteiligt und zählen zu den Verdächtigen. Auch unabhängig vom Kriminalfall stehen sie im Kontext von Problemen wie Gettoisierung oder gewalttätigen Konflikten als Folge misslungener Integration. Die einheitliche, eher negative Darstellung der Migrantenfiguren, die nur durch einen türkischstämmigen Kollegen von Schenk und Ballauf durchbrochen wird, scheint wenig geeignet, zum Abbau von Vorurteilen beizutragen.

Zudem bekommen die Zuseher kaum Gelegenheit, den Konflikt aus der Sichtweise der Migrantenfiguren zu beurteilen, denn die türkischen Schüler äußern sich nur selten zu den Ereignissen. Ihr Verhalten lässt sich schwer nachvollziehen, da die Zuschauer kaum Informationen über ihre Probleme, Ängste und ihr Lebensumfeld erhalten. Insgesamt liegt der Fokus des Films deutlich auf den deutschen Protagonisten.

Da die Feindschaft zwischen den Schülern zentrales Thema des Films ist, werden In- und Ausländer stark in Opposition zueinander dargestellt. Das Zugehörigkeitsgefühl zur Nation der Eltern ist sowohl für die deutschen als auch für die türkischen Schüler identitätsstiftend und führt zu Gruppenbildung. Diese Einteilung ist jedoch stark konstruiert, denn abgesehen von der Herkunft gibt es mehr Gemeinsamkeiten als Unterschiede. Ballauf weist einen der Schüler explizit auf Ähnlichkeiten hin und Schenk hinterfragt das Zugehörigkeitsgefühl der türkischstämmigen Kinder zur Türkei. Anders als die Schüler zieht er nicht die Herkunft der Eltern, sondern das Geburtsland als Merkmal für nationale Zugehörigkeit heran, wodurch deutlich wird, dass die Grenze zwischen In- und Ausländern individuell verschieden gezogen werden kann.

Betrachtet man die Umgangsweisen zwischen Deutschen und Migranten in Kinder der Gewalt, so wird deutlich, dass sich der Film weitgehend auf die Darstellung von Problemen beschränkt. Im Vordergrund steht das negative Beispiel der verfeindeten Schüler, und da keine der Figuren eine Entwicklung oder einen Lernprozess durchläuft, mangelt es an Lösungsansätzen für den Konflikt.

16 Bei der ersten Ausstrahlung am 2.5.1999 erreichte Kinder der Gewalt 7,88 Mio. Zuseher und damit einen Marktanteil von 24,04 \%. Danach wurde die Folge noch vier Mal ausgestrahlt. (Tatort-Fundus, 2006) 


\section{Reise ins Nichts ${ }^{17}$ (SR 2002, Palü)}

In der Folge Reise ins Nichts wird eine Gruppe Afghanen von Schleppern nach Deutschland gebracht. Dabei kommt es zu einem Zwischenfall, bei dem zwei von ihnen sterben und ein Mädchen von der Gruppe getrennt wird.

Im Gegensatz zu den anderen analysierten Filmen bietet diese Folge den Zusehern kaum Anregungen zur Auseinandersetzung mit Vorurteilen, denn Fremdenfeindlichkeit spielt eine geringe Rolle. Da Kommissar Palü nicht mit ausländerfeindlichen Aussagen konfrontiert wird, äußert er sich auch nicht zu diesem Thema. Auch die Art und Weise, wie die Migrantenfiguren dargestellt werden, hat wenig Potenzial, zum Abbau stereotyper Vorstellungen beizutragen. Die Einwanderer treten großteils in der Gruppe auf, sehen einander zum Verwechseln ähnlich und entsprechen gängigen Klischeevorstellungen. Sie sind in verschlissene Gewänder gehüllt, haben dunkle Haare und Augen, die Frauen tragen Kopftücher, die Männer Turban oder Gebetskappe. Zudem werden die Flüchtlinge in erster Linie als namenlose Opfer ohne Handlungsspielraum dargestellt. Nur Khandi bekommt im Laufe des Films individuelle Züge; ausführlich charakterisiert wird zudem noch Habib, der mit einem früheren Transport nach Deutschland gebracht wurde. Für kurze Zeit steht er unter Tatverdacht; allerdings stellt sich sehr bald heraus, dass er unschuldig ist.

Auf Grund mangelnder Sprachkenntnisse können die Einwanderer nicht von ihren Erlebnissen erzählen. An mehreren Stellen werden allerdings Informationen zu Menschenschmuggel eingeflochten, die bei den deutschen Figuren Mitgefühl auslösen. Es handelt sich dabei um abstrakte Zahlen, die nur bedingt zum Verständnis der Flüchtlinge beitragen können, denn sie sagen nichts darüber aus, warum die Einwanderer geflohen sind, wie sie die Reise erlebt haben oder welche Konsequenzen eine Abschiebung für sie hätte. Im Gegensatz zu den Migranten artikulieren die deutschen Figuren wiederholt ihre Sichtweise und begründen damit ihr Verhalten. Dies trifft sogar auf den Schlepper Hallmeier zu: Er hat den Menschenschmuggel begonnen, weil er Geld braucht, um seine Spedition vor dem Ruin zu retten, und rechtfertigt sich damit, dass er den Einwanderern zu einem besseren Leben verhelfe.

Als Folge der reduzierten Figurenzeichnung der Migranten lassen sich die Figuren in Reise ins Nichts problemlos in In- und Ausländer unterteilen. Dies gilt auch für Khandi und Habib, obgleich sie ausführlicher charakterisiert werden. Die Frage nach der Zugehörigkeit der Flüchtlinge zur Aufnahmegesellschaft wird in Reise ins Nichts nicht gestellt, da von Beginn an fest steht, dass sie wieder abgeschoben werden, sobald die Polizei ihr Versteck ausfindig gemacht hat.

Dessen ungeachtet knüpfen einige der Migranten im Einwanderungsland schnell Kontakte und pflegen enge Beziehungen zu Deutschen. Kommissar Palü und seine Frau Margit nehmen Khandi bei sich zu Hause auf und entwickeln innerhalb weniger Tage eine vertraute Beziehung zu dem Mädchen. Habib, der sich schon eine Weile in Deutschland aufhält, ist mit Hallmeiers Tochter Sabine liiert. Am Ende des Films zeigen sich Palü und seine Frau solidarisch mit den Flüchtlingen und kritisieren die strengen Gesetze, die zu ihrer Abschiebung führen. An positiven Modellen des gegenseitigen Umgangs mangelt es in Reise ins Nichts folglich nicht.

17 Bei der Erstsendung am 29.12.2002 sahen 6,52 Mio. Menschen die Folge Reise ins Nichts; der Marktanteil betrug 19,20\%. Seither gab es vier Wiederholungen. (Tatort-Fundus, 2006) 


\section{Fazit}

Vor dem Hintergrund integrationsfördernder Potenziale fiktionaler Fernsehinhalte hat sich der Beitrag mit der Thematisierung von Migration in der Krimireihe Tatort auseinandergesetzt. Dabei wurde deutlich, dass Einwanderung im Tatort einen wichtigen Platz einnimmt und im Laufe der Jahre an Bedeutung gewonnen hat. Von den insgesamt 566 Episoden wird in zumindest 92 Migration angesprochen, in 32 davon steht das Thema im Mittelpunkt des Films. Da Tatort seit den 70er Jahren zu den quotenstärksten fiktionalen TV-Angeboten zählt, bringt die Reihe das Thema Einwanderung einem Massenpublikum auf unterhaltsame Weise näher. Insgesamt umfassen die 32 Folgen mit Migration als Hauptthema 14 unterschiedliche Teilaspekte; auffälligerweise handelt es sich dabei häufig um problembehaftete Kontexte.

Die qualitativen Einzelanalysen von fünf ausgewählten Episoden zeigen exemplarisch, dass Tatort-Filme das Oberthema Migration weitgehend facettenreich umsetzen. Potenziell integrationsfördernde Merkmale lassen sich dennoch nur in eingeschränktem Ausmaß feststellen. Am intensivsten nutzen die analysierten Folgen die Möglichkeit, positive Modelle des gegenseitigen Umgangs von Menschen unterschiedlicher Herkunft aufzuzeigen, die den Zusehern als Orientierungsrahmen für das eigene Handeln dienen können. Darüber hinaus bieten sie Anregungen zur Beschäftigung mit Fremdenfeindlichkeit und Vorurteilen. Betrachtet man die Darstellung der Migrantenfiguren, so scheinen die Filme dennoch nur bedingt geeignet, zum Abbau von Vorurteilen beizutragen. Obwohl der überwiegende Teil der Einwanderer eher positive Eigenschaften aufweist, kommen in allen analysierten Tatort-Episoden zumindest am Rande klischeebehaftete und negative Figurenzeichnungen vor. Da den fünf Folgen mit einer Ausnahme eine klare Einteilung der Figuren entlang ihrer Herkunft zugrunde liegt, haben sie überdies wenig Potenzial, die übliche Grenzziehung zwischen den beiden Gruppen In- und Ausländer aufzuweichen und so die Wahrnehmung von Migranten als Teil der Gesellschaft zu fördern. Zudem dominieren die deutschen Figuren die Erzählungen. Sie kommen nicht nur häufiger in Hauptrollen vor, die Zuseher erfahren auch mehr über ihr Privatleben, ihren Alltag und ihr berufliches Umfeld. Nur die Folge In der Falle stellt Migranten in das Zentrum der Erzählung, weist eine Reihe an Identifikationsfiguren anderer Herkunft auf und ermöglicht den Zuschauern, die Ereignisse aus dem individuellen Blickwinkel von Einwanderern zu sehen.

Zusammenfassend lässt sich festhalten, dass die Krimireihe Tatort einen wichtigen Beitrag zur Diskussion von Einwanderung in den Medien leistet. Dennoch scheint das inhaltliche Angebot der Reihe nur bedingt geeignet, stereotype Vorstellungen zu durchbrechen, Vorurteilen entgegenzuwirken oder die Wahrnehmung von Migranten als Teil der Gesellschaft zu fördern. Um der Frage nach möglichen integrationsfördernden Einflüssen fiktionaler TV-Inhalte wie Tatort weiter nachzugehen, wären in einem zweiten Schritt Untersuchungen zur Rezeption und Aneignung solcher Angebote nötig. Lebensweltliche Kontexte sollten dabei ebenso berücksichtigt werden wie Faktoren, die nach den Erkenntnissen der Vorurteilsforschung zur Meinungsbildung über Migranten, zur Herausbildung von Abgrenzungsmechanismen sowie zur Ausprägung von Verhaltensmustern beitragen. Nur so lässt sich feststellen, ob und unter welchen Bedingungen die identifizierten Potenziale fiktionaler TV-Inhalte tatsächlich zum Tragen kommen. 


\section{Literatur}

Bauer, Ludwig (1992): Authentizität, Mimesis, Fiktion. Fernsehunterhaltung und Integration von Realität am Beispiel des Kriminalsujets. Diskurs Film: Bibliothek, Bd. 3. München: Schaudig, Bauer, Ledig.

Berghaus, Margot (1999): Wie Massenmedien wirken. Ein Modell zur Systematisierung. In: Rundfunk und Fernsehen, 2/1999, S. 181-199.

Brosius, Hans-Bernd (2003): Medienwirkung. In: Bentele, Günter/Brosius, Hans-Bernd/Jarren, Otfried (Hrsg.): Öffentliche Kommunikation. Handbuch Kommunikations- und Medienwissenschaft. Wiesbaden: Westdeutscher Verlag, S. 128-148.

Brosius, Hans-Bernd/Esser, Frank (1995): Fernsehen als Brandstifter? Unerwünschte Nebenwirkungen der Berichterstattung über fremdenfeindliche Gewalt. In: Falter, Jürgen W./Jaschke, Hans-Gerd/Winkler, Jürgen R. (Hrsg.): Rechtsextremismus. Ergebnisse und Perspektiven der Forschung. Opladen: Westdeutscher Verlag, S. 204-218.

Brück, Ingrid/Viehoff, Reinhold (1998): Crime genre and television. From Stahlnetz to Tatort. A realistic tradition. In: Viehoff, Reinhold (Hrsg.): Stahlnetz, Tatort, Polizeiruf 110. Transitions in German Police Series. HALMA - Hallische Medienarbeiten 8, S. 3-11.

Brück, Ingrid/Guder, Andrea/Viehoff, Reinhold/Wehn, Karin (2003): Der deutsche Fernsehkrimi. Eine Programm- und Produktionsgeschichte von den Anfängen bis heute. Stuttgart/Weimar: Metzler.

Bulut, Claudia (2000): Von der Gastarbeiterin zur Schutzpolizistin. Das konstruierte Bild der fremden Frau im deutschen Film und Fernsehen. In: Schatz, Heribert/Holtz-Bacha, Christina/Nieland, Jörg-Uwe (Hrsg.): Migranten und Medien. Neue Herausforderungen an die Integrationsfunktion von Presse und Rundfunk. Wiesbaden: Westdeutscher Verlag, S. 253-264.

Darschin, Wolfgang/Gerhard, Heinz (2004): Tendenzen im Zuschauer-Verhalten. Fernsehgewohnheiten und Fernsehreichweiten im Jahr 2003. In: Media Perspektiven 4/2004, S. 142-150.

Delgado, Jesus M. (1972): Die „Gastarbeiter“ in der Presse. Eine inhaltsanalytische Studie. Opladen: Leske.

Dörner, Andreas (2000): Das Kino als politische Integrationsagentur. Afroamerikanische Identitätsangebote im Hollywood-Film der 90er Jahre. In: Schatz, Heribert/Holtz-Bacha, Christina/Nieland, Jörg-Uwe (Hrsg.): Migranten und Medien. Neue Herausforderungen an die Integrationsfunktion von Presse und Rundfunk. Wiesbaden: Westdeutscher Verlag, S. 164-180.

Eckhardt, Josef (1987): Ausländerthematische Fernsehsendungen und deutsche Zuseher. In: Media Perspektiven 5/1987, S. 311-316.

Esser, Hartmut (1986): Social context and interethnic relations. The case of migrant workers in West German urban areas. In: European Socialogical Review 2/1986, S. 30-51.

Esser, Hartmut (2000): Assimilation, Integration und ethnische Konflikte. Können sie durch „Kommunikation“ beeinflußt werden? In: Schatz, Heribert/Holtz-Bacha, Christina/Nieland, Jörg-Uwe (Hrsg.): Migranten und Medien. Neue Herausforderungen an die Integrationsfunktion von Presse und Rundfunk. Wiesbaden: Westdeutscher Verlag, S. 25-37.

Geißler, Rainer/Pöttker, Horst (2005): Bilanz. In: Geißler, Rainer/Pöttker, Horst (Hrsg.): Massenmedien und die Integration ethnischer Minderheiten in Deutschland. Problemaufriss - Forschungsstand - Bibliographie. Bielefeld: transcript Verlag, S. 391-396.

Gerhards, Maria; Klingler, Walter (2005): Programmangebote und Spartennutzung im Fernsehen. Ergebnisse 2004 und ein Fünfjahresvergleich auf Basis der AGF/GfK-Programmcodierung. In: Media Perspektiven 11/2005, S. 588-569.

Güven, Dilec (1996): Das (Heirats-)Versprechen auf der (Polizei-)Wache. Stereotypen über Türken in deutschen TV-Serien. Köln: Media Watch.

Hickethier, Knut (1991): Die Fernsehserie und das Serielle des Fernsehens. Kultur, Medien, Kommunikation. Lüneburger Beiträge zur Kulturwissenschaft, Bd. 2.

Jäger, Siegfried (2000): Von deutschen Einzeltätern und ausländischen Banden. Medien und Straftaten. In: Schatz, Heribert/Holtz-Bacha, Christina/Nieland, Jörg-Uwe (Hrsg.): Migranten und Medien. Neue Herausforderungen an die Integrationsfunktion von Presse und Rundfunk. Wiesbaden: Westdeutscher Verlag, S. 207-216. 
Jarren, Otfried (2000): Gesellschaftliche Integration durch Medien? Zur Begründung normativer Anforderungen an Medien. In: Medien \& Kommunikationswissenschaft 1/2000, S. 22-41.

Kade, Jochen (1996): „Tatort“ und „Polizeiruf 110“ zur biographischen Kommunikation des Fernsehens in beiden deutschen Staaten. In: BIOS - Zeitschrift für Biographieforschung und Oral History, 1/1996, S. 114-126.

Klaus, Elisabeth/Goldbeck, Kerstin/Kassel, Susanne (2002): Fremd- und Selbstbilder in der Berichterstattung der deutschen Medien während des Kosovokrieges - am Beispiel des Spiegel. In: Imhof, Kurt/Jarren, Otfried/Blum, Roger (Hrsg.): Integration und Medien. Mediensymposium Luzern, Bd. 7. Wiesbaden: Westdeutscher Verlag, S. 285-305.

Krüger, Udo M./Simon, Erik (2005): Das Bild der Migranten im WDR Fernsehen. Ergebnisse einer empirischen Programmanalyse. In: Media Perspektiven 3/2005, S. 105-114.

Kühne-Scholand, Hildegard (1987): Die Darstellung der Ausländer im deutschen Fernsehen. Ergebnisse einer inhaltsanalytischen Untersuchung. In: Bundeszentrale für politische Bildung (Hrsg.): Ausländer und Massenmedien. Bestandsaufnahme und Perspektiven. Vorträge und Materialien einer internationalen Fachtagung vom 2. bis 4. Dezember 1986. Bonn: Bundeszentrale für politische Bildung, S. 79-84.

Liebhart, Karin/Menasse, Elisabeth/Steinert, Heinz (2002): Fremdbilder - Feindbilder - Zerrbilder. Zur Wahrnehmung und diskursiven Konstruktion des Fremden. In: Liebhart, Karin/Menasse, Elisabeth/Steinert, Heinz (Hrsg.): Fremdbilder - Feindbilder - Zerrbilder. Zur Wahrnehmung und diskursiven Konstruktion des Fremden. Publikationsreihe des Bundesministeriums für Bildung, Wissenschaft und Kultur zum Forschungsschwerpunkt Fremdenfeindlichkeit, Bd. 5. Klagenfurt: Drava-Verlag, S. 7-14.

Meißner, Bettina/Ruhrmann, Georg (2000): Das Ausländerbild in den Thüringer Tageszeitungen. Eine quantitative und qualitative Inhaltsanalyse. Jena: Friedrich-Schiller-Universität 2001.

Merten, Klaus (1986): Das Bild der Ausländer in der deutschen Presse. Ergebnisse einer systematischen Inhaltsanalyse. Frankfurt am Main: Dağyeli.

Mikos, Lothar (1987): Fernsehserien. Ihre Geschichte, Erzählweise und Themen. In: Medien und Erziehung 1/1987, S. 2-16.

Müller, Daniel (2005): Die Darstellung ethnischer Minderheiten in deutschen Massenmedien. In: Geißler, Rainer/Pöttker, Horst (Hrsg.): Massenmedien und die Integration ethnischer Minderheiten in Deutschland. Problemaufriss, Forschungsstand, Bibliographie. Bielefeld: transcript Verlag, S. 84-126.

Rothmund, Jutta/Schreier, Margrit/Groeben, Norbert (2001): Fernsehen und erlebte Wirklichkeit II. Ein integratives Modell zu Realitäts-Fiktions-Unterscheidungen bei der (kompetenten) Mediennutzung. In: Zeitschrift für Medienpsychologie, 2/2001, S. 85-95.

Ruhrmann, Georg/Kollmer, Jochen (1987): Ausländerberichterstattung in der Kommune. Inhaltsanalyse Bielefelder Tageszeitungen unter besonderer Berücksichtigung „ausländerfeindlicher" Alltagstheorien. Opladen: Westdeutscher Verlag.

Ruhrmann, Georg/Sommer, Denise/Uhlemann, Heike (2006): TV-Nachrichtenberichterstattung über Migranten. Von der Politik zum Terror. In: Geißler, Rainer/Pöttker, Horst (Hrsg.): Integration durch Massenmedien. Mass Media-Integration. Medien und Migranten im internationalen Vergleich. Media and Migration: A Comparative Perspective. Medienumbrüche 17. Bielefeld: transcript Verlag, S. 45-75.

Schenk, Michael (2002): Medienwirkungsforschung. 2., vollständig überarbeitete Auflage. Tübingen: Mohr Siebeck.

Schnake, Karin (2000): „Klinik unter Palmen“ und „Tatort“. Information als Affektträger von Unterhaltung. In: Paus-Haase, Ingrid/Schnatmeyer, Dorothee/Wegener, Claudia (Hrsg.): Information, Emotion, Sensation. Wenn im Fernsehen die Grenzen zerfließen. Schriften zur Medienpädagogik, Bd. 30. Bielefeld: GMK, S. 210-233.

Schorb, Bernd/Echtermeyer, Karin/Lauber, Achim/Eggert, Susanne (2003): Was guckst du, was denkst du? Der Einfluss des Fernsehens auf das Ausländerbild von Kindern im Alter von 9 bis 14 Jahren. Schriftenreihe der Unabhängigen Landesanstalt für Rundfunk und neue Medien (ULR), Bd. 22. Kiel: Schmidt \& Klaunig.

Süss, Daniel (1993): Der Fernsehkrimi, sein Autor und die jugendlichen Zuschauer. Medien- 
kommunikation aus drei Perspektiven, am Beispiel des „Tatort“-Krimis „Kameraden“. Bern: Huber.

Tatort-Fundus (2006): Die private Internetseite zum Krimi-Klassiker von ARD, ORF (und SFDRS). Verfügbar unter: http://www.tatort-fundus.de [Stand 12.01.2007].

Thiele, Matthias (2005): Flucht, Asyl und Einwanderung im Fernsehen. Konstanz: UVK Verlagsgesellschaft.

Vogt, Jochen (2004): Tatort - der wahre deutsche Gesellschaftsroman. Eine Projektskizze. In:

Vogt, Jochen (Hrsg.): MedienMorde. Krimis intermedial. Paderborn: Fink Verlag, S. 111-129.

Volf, Patrik/Bauböck, Rainer (2001): Wege zur Integration. Was man gegen Diskriminierung und Fremdenfeindlichkeit tun kann. Publikationsreihe des Bundesministeriums für Bildung, Wissenschaft und Kultur zum Forschungsschwerpunkt Fremdenfeindlichkeit, Bd. 4. Klagenfurt/Celovec: Drava-Verlag.

Weber-Menges, Sonja (2005): Die Wirkungen der Präsentation ethnischer Minderheiten in deutschen Medien. In: Geißler, Rainer/Pöttker, Horst (Hrsg.): Massenmedien und die Integration ethnischer Minderheiten in Deutschland. Problemaufriss, Forschungsstand, Bibliographie. Bielefeld: transcript Verlag, S. 127-184.

Wehn, Karin (2002): ,Crime-Time‘ im Wandel. Produktion, Vermittlung und Genreentwicklung des west- und ostdeutschen Fernsehkrimis im Dualen Rundfunksystem. Bonn: ARCult Media (Reihe MedienKultur; 3).

Weiß, Hans-Jürgen/Feike, Martina/Freese, Walter/Funk, Peter/Trebbe, Joachim (1995): Gewalt von Rechts - (k)ein Fernsehthema? Zur Fernsehberichterstattung über Rechtsextremismus, Ausländer und Asyl in Deutschland. Opladen: Leske + Budrich. 